\title{
Tuberculoid relapse in lepromatous leprosy
}

\author{
M F R WATERS \& D S RIDLEY \\ Hospital for Tropical Diseases, London NW1 OPE and The School \\ of Pathology, University College and Middlesex School of Medicine, \\ Ridinghouse Street, London WIP 7PN
}

Accepted for publication 2 May 1990

\begin{abstract}
Summary It is commonly accepted that the attainment of bacteriological negativity fails to restore the immune state of leprosy patients who have downgraded to lepromatous. We report six patients who had been lepromatous (LLs), and who, after many years of chemotherapy and bacteriological negativity, were found upon relapse to have upgraded to borderline-tuberculoid (BT). Five had become Mitsuda lepromin positive. The relapses could be accounted for by proven or suspected dapsone resistance. The upgrading was associated with minimal signs of reaction, which was attributed to the low level of antigen in the almost resolved lesions. The manner of development of the new high immune lesions resembled the onset of a primary infection, clinically and histologically. The development of a positive Mitsuda reaction in longstanding LL leprosy is not necessarily an indication of cure.
\end{abstract}

\section{Introduction}

Before the advent of multidrug therapy (MDT), when long-treated, smear negative lepromatous patients relapsed, from whatever cause, their relapse lesions almost invariably retained lepromatous (LL) or borderline-lepromatous (BL) characteristics, and they remained lepromin negative. ${ }^{1,2}$ Occasional $\mathrm{BL}$ patients have relapsed as borderline-tuberculoid (BT), but usually as a consequence of reversal (upgrading, Jopling type 1) reactions during treatment. In 1978, we reported ${ }^{3}$ for the first time three subpolar lepromatous ${ }^{4}$ (LLs) patients who had relapsed with BT leprosy without having undergone any marked reaction. We report here three further patients, together with a follow up of our original cases. In addition, we have performed control lepromin studies, with unexpected findings, which we report in an associated paper. ${ }^{5}$

\section{Case reports}

1 PATIENT 5077

This Chinese patient was admitted to Sungai Buluh Leprosarium, Malaysia, in 1937, 


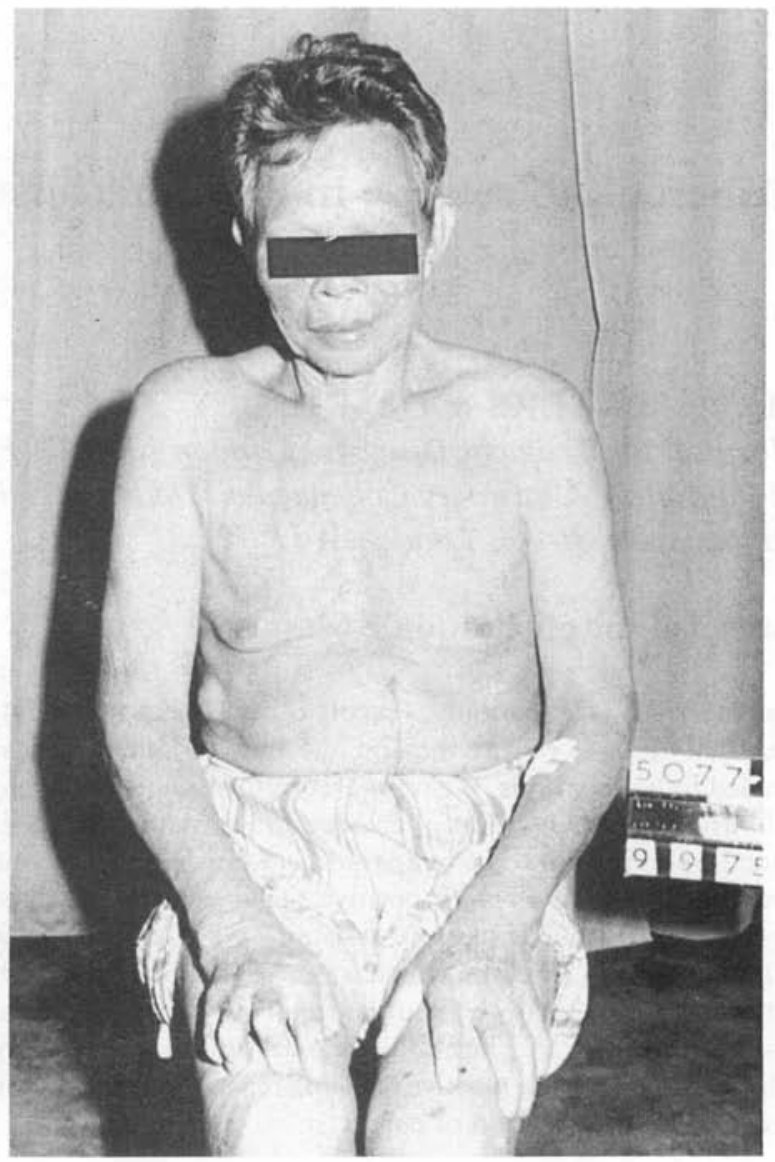

Figure 1. Patient No. 1. Front view showing the typical features of well-treated, advanced LL leprosy. There is clawing of the right hand.

when aged 35. In 1947, he was graded 'L3' with failing vision due to lepromatous infiltration and iritis. He was treated with dapsone from 1948 to 1970 . His smears were negative from 1959 to 1974.

Annual examinations were satisfactory until 1975, when erythematous patches were noted on his trunk. On referral to the Leprosy Research Unit three months later, ${ }^{6}$ his general appearance was considered consistent with well-treated, advanced LLs leprosy (Figure 1). The skin was wrinkled, madarosis was almost complete, nerves were enlarged and there was widespread anaesthesia over the limbs and trunk, with a right ulnar and median claw hand. Yet there were fading erythematous raised annular tuberculoid lesions on both flanks, two on the left and three on the right (Figure 2). Smears from these lesions gave a bacterial index $(\mathrm{BI})$ on the Ridley scale of $1+$; elsewhere they were negative. The Mitsuda reaction (standard Wade-Mitsuda lepromin) measured $9 \mathrm{~mm}$ at three weeks and $6 \mathrm{~mm}$ with ulceration at four weeks. Two annular lesions were biopsied, revealing a rather nondescript mass of lymphocytes. A year later, after parenteral dapsone, $400 \mathrm{mg}$ twice weekly, he was graded histologically as BT (Figures 3 and 4). 


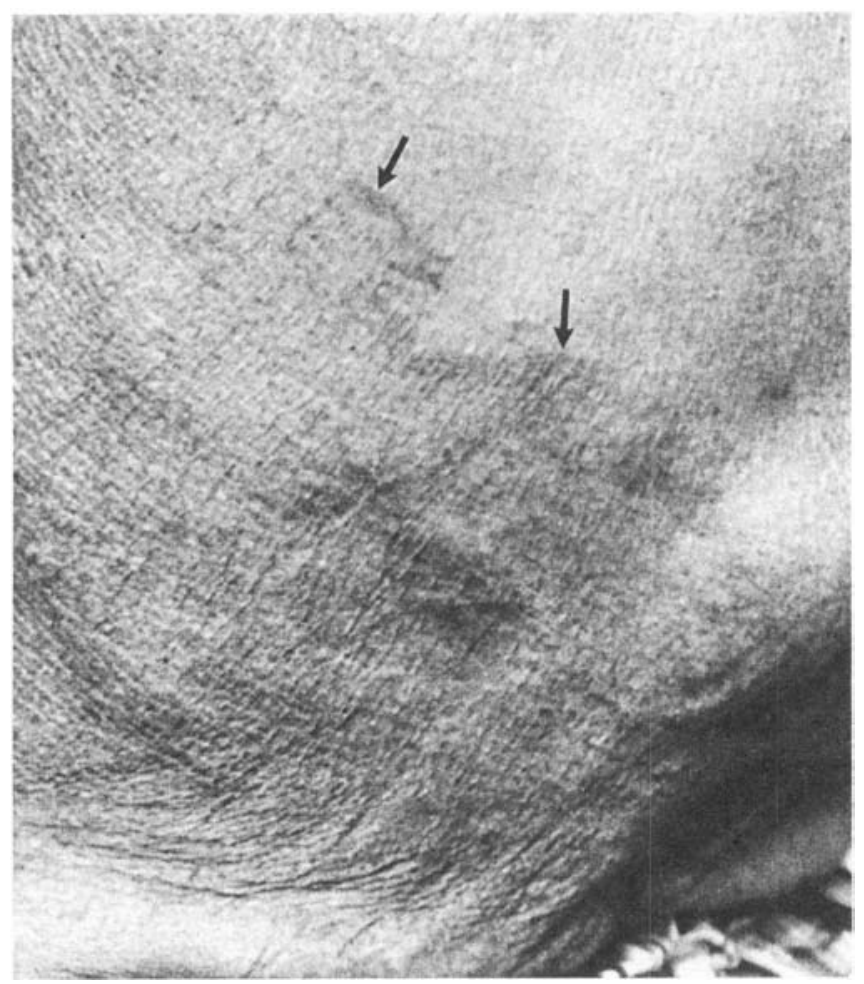

Figure 2. Patient No. 1. Right flank showing (arrowed) irregular-edged, annular lesions typical of BT leprosy.

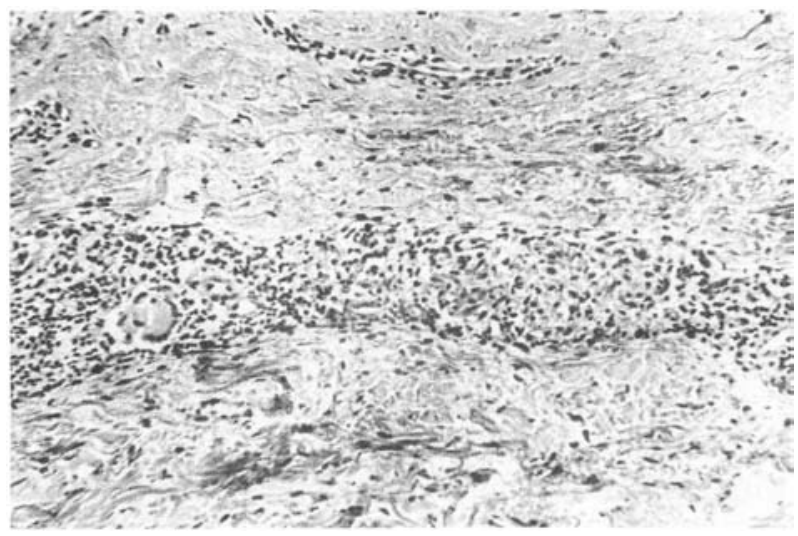

Figure 3. Patient No. 1. Biopsy of annular lesion, taken one year after recommencing dapsone therapy. The appearances are of a BT leprosy granuloma with many lymphocytes.

From 1976 to 1981, chemotherapy was continued unchanged owing to severe gastric ulcer symptoms, with little change in his condition save for some fluctuation in the degree of erythema and induration in the annular edges of the lesions. Repeat skin biopsy in 1979 again revealed the histological appearances of BT leprosy with mild reaction but no signs 


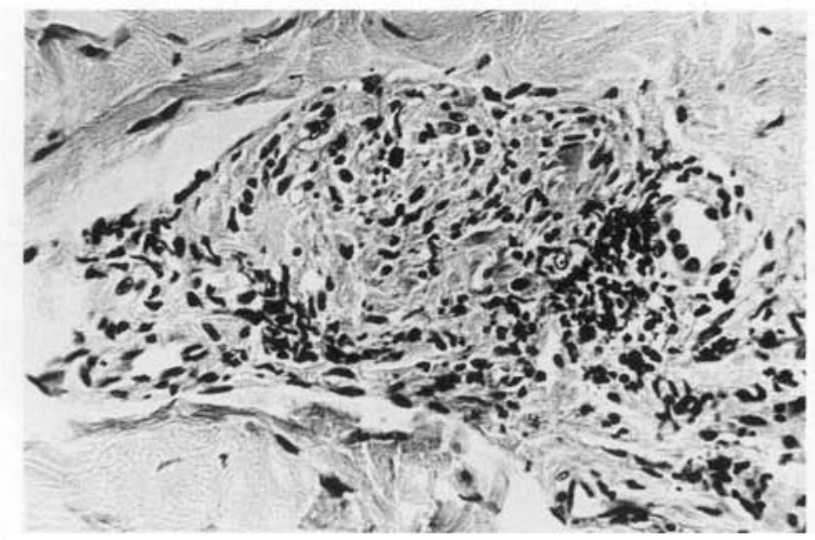

Figure 4. Patient No. 1. Infiltration and disorganization of a dermal nerve. From the same lesion as Figure 3.

of downgrading or active spread; a few granular acid-fast bacilli (AFB) were noted. A fresh biopsy homogenate yielded a BI of $3+$ with a morphological index of 10 . Mouse footpad inoculation showed the strain to be fully resistant to dapsone (at $0.01 \%$ concentration in the mouse diet) but sensitive to thiambutosine (at $0 \cdot 1 \%$ concentration). A repeat lepromin test in 1980 was still strongly positive, and skin smears from his relapse lesions were negative.

\section{PATIENT 8715}

This male Chinese patient was admitted to Pulau Jerejak Leprosarium, Malaysia, in 1939 when aged about 16 years. His voice became hoarse in 1945, and his right eye blind in 1946. He commenced treatment with dapsone around 1950 and became skin smearnegative around 1960. He was transferred to Sungai Buluh Leprosarium in 1969, and remained smear negative until June 1974, when new lesions were observed.

When he was referred to the Leprosy Research Unit in May 1975, his clinical appearance was consistent with treated, advanced LLs leprosy, with 'rat-bitten ears', madarosis and a deformed nose and soft palate. Nerves were slightly and symmetrically enlarged. New erythematous, dryish papules and plaques with irregular edges were present on the arms, trunk and lower limbs (Figure 5). One lesion on the right thigh was more borderline with a hollowed-out centre. His smears gave a BI of 1.2; the Mitsuda reaction measured $5 \mathrm{~mm}$ at four weeks and histological examination of lesions on the back and arm revealed $\mathrm{BT}$ in reaction.

On treatment with dapsone $400 \mathrm{mg}$ by injection twice weekly, he initially improved. But 15 months later further new lesions were more lepromatous in character and histologically BL. His strain of Mycobacterium leprae was shown to be fully resistant to dapsone $(0.01 \%$ concentration in the mouse diet $)$.

\section{PATIENT RS}

A 60-year-old Punjabi was referred to Dr Jopling in 1966, complaining of epistaxes and swelling of the ankles. On examination (Figure 6), there was widespread diffuse 


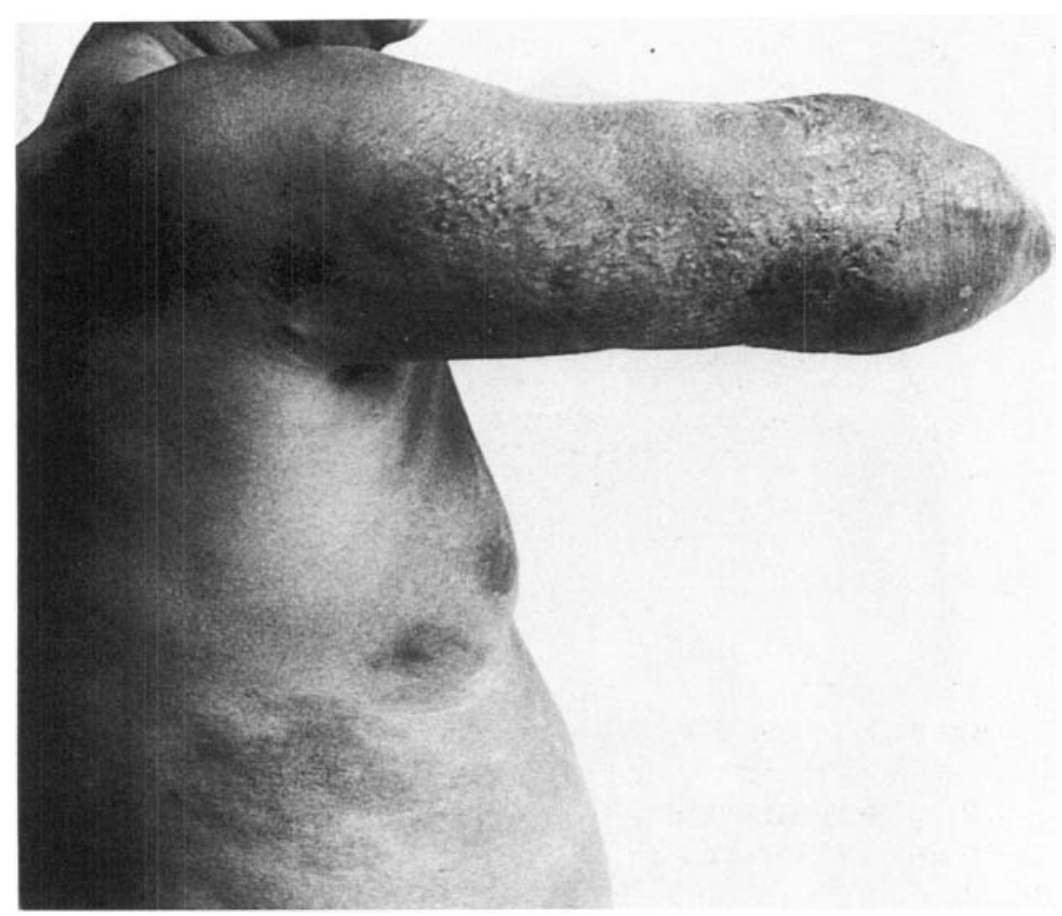

Figure 5. Patient No. 2. Right arm and chest showing erythematous plaques (on chest) and coalescing papules (on arm), suggestive of BT or BT/BB leprosy.

lepromatous infiltration with some mild erythema nodosum leprosum (ENL), complete madarosis, mild symmetrical nerve enlargement, and anaesthesia of the feet and ankles. The lepromin test was negative and the smear BI 4.2. Histologically, he was graded as typical LLs (Figure 7), which was mildly active with low grade ENL. He responded well to treatment with dapsone (plus Vadrine for the first six months) becoming smear negative in 1973. In 1975, a biopsy showed an infiltrative, mainly lymphocytic lesion which was now almost indeterminate but with some tuberculoid features: erosion of the epidermis and destruction of sweat glands. There was also mild reactional oedema, and one short solidstaining bacillus. He was smear negative in May 1977, when clinically he resembled welltreated, inactive, polar or near-polar lepromatous leprosy. He admitted to taking little if any dapsone during the previous six months owing to chronic bronchitis.

Three months later he was found to have developed erythematous, raised, annular lesions in both lumbar regions. Histologically, the lesions were now BT with many lymphocytes and not far removed from TT (Figure 8). There was no active spread and no AFB; smears were negative. His Fernandez reaction measured $5 \mathrm{~mm}$ at 3 days; no Mitsuda reading was then possible. The lesions subsided steadily on dapsone $50 \mathrm{mg}$ daily. After one year they were virtually invisible, though smears were $1+$ positive. A repeat lepromin test in October 1978 gave a Mitsuda reading of $3 \mathrm{~mm}$ at 4 weeks. In 1979, his treatment was changed to clofazimine, with rifampicin initially, for fear of dapsone - resistance. Three of six mouse footpads inoculated with a homogenate from a new lesion 


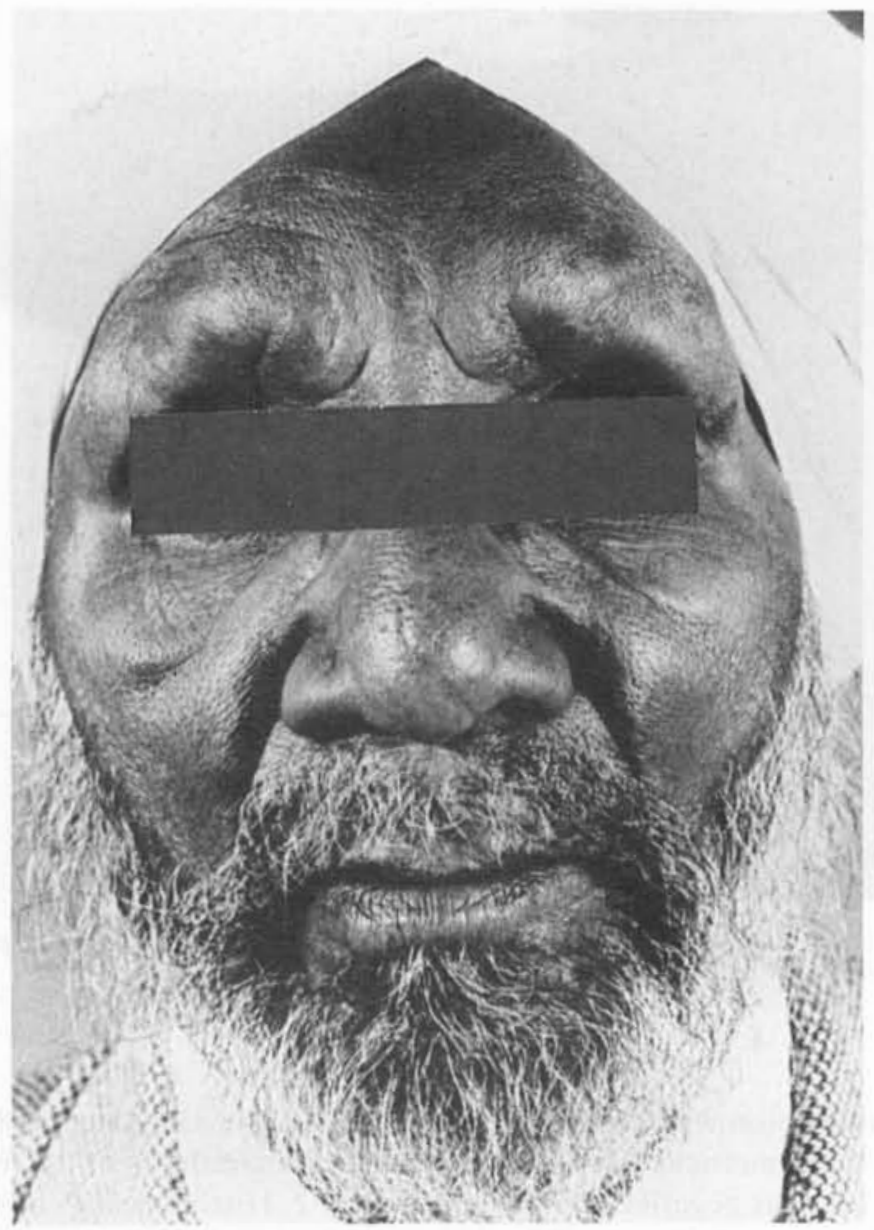

Figure 6. Patient No. 3. View of face, on first diagnosis in 1966, showing typical appearances of advanced untreated LL leprosy.

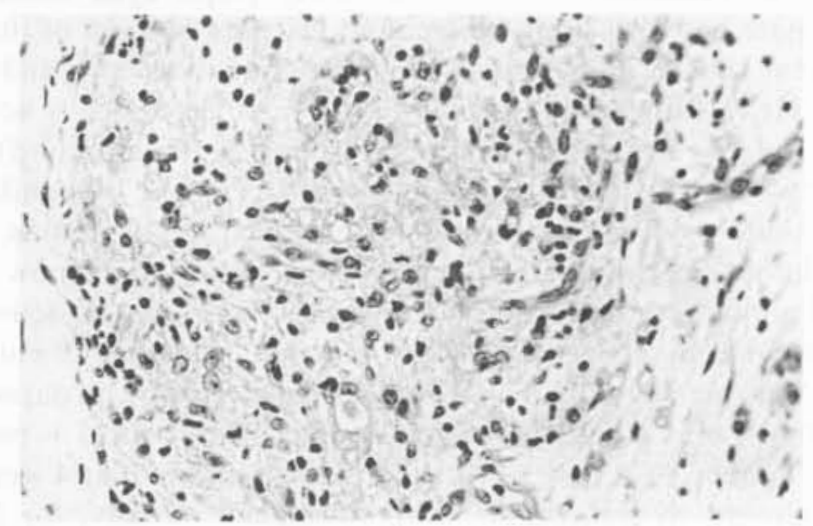

Figure 7. Patient No. 3. Histological appearances of skin on first diagnosis in 1966, showing mildly active LLs leprosy. 


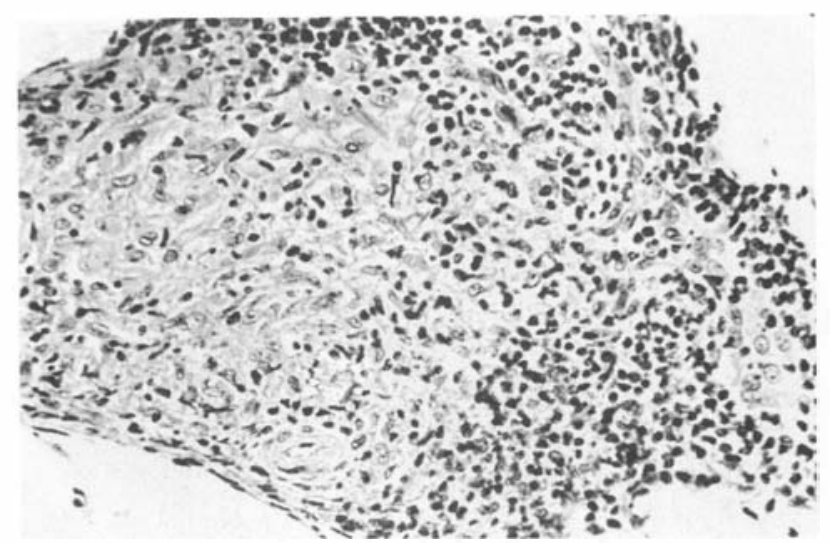

Figure 8. Patient No. 3. Histology of a relapse skin lesion in 1977; the appearances are of BT leprosy with many lymphocytes, not far removed from TT.

taken in August 1977 had become positive for $M$. leprae, and subsequent passage confirmed full dose dapsone resistance. He died of intercurrent disease in 1980.

\section{PATIENT TB}

This Anglo-Indian patient, aged 25, developed a severe ulcerating ENL reaction, progressive deformity of his fingers and hands and left foot drop, following treatment with sulphoxone sodium (Diasone) in 1950. Chemotherapy was changed to thiacetazone, and then stopped altogether in 1953. The ENL ceased around the same time. He arrived in England and was admitted to the Hospital for Tropical Diseases in 1954. In addition to the deformities noted above, he had 'rat-bitten' ears and a large nasal septal perforation, clawed toes, and numerous whitish scars chiefly on his limbs, compatible with past severe ulcerations. There was widespread enlargement of nerves. His eyebrows were intact. Skin smears were weakly positive for AFB (overall BI 1.0, with the densest site $3+$ ). The Mitsuda reaction was negative. Histologically, his skin biopsy was classified as LLs; the lesion was small but with some solid AFB. It was considered that his treatment in 1950 had commenced shortly after downgrading from borderline to lepromatous. He was restarted on dapsone and within a month episodes of mild ENL recurred and these continued for about five months. After that the lesions regressed, and he became skinsmear negative by November 1956. He stopped taking dapsone in 1962, but recommenced in 1963, because of bilateral iridocyclitis, for which he received corticosteroids almost continuously for the next 15 years. From 1965 to 1970, he received low dose thiambutosine and then restarted dapsone, the dose being raised to $100 \mathrm{mg}$ daily in 1977. His urine was repeatedly positive for dapsone. In August 1978, the lepromatous leprosy was clinically completely quiescent and his smears (6 sites) were negative for AFB.

Two months later he developed erythematous plaques over his right forehead and his face, which increased slightly in size; their edges were indurated and raised (Figure 9). Clinically they were BT. Histologically, a lesion was characterized by many lymphocytes and undifferentiated histiocytic cells, but no granuloma or AFB; it was classified as indeterminate, but tuberculoid rather than lepromatous. Smears were negative. The 


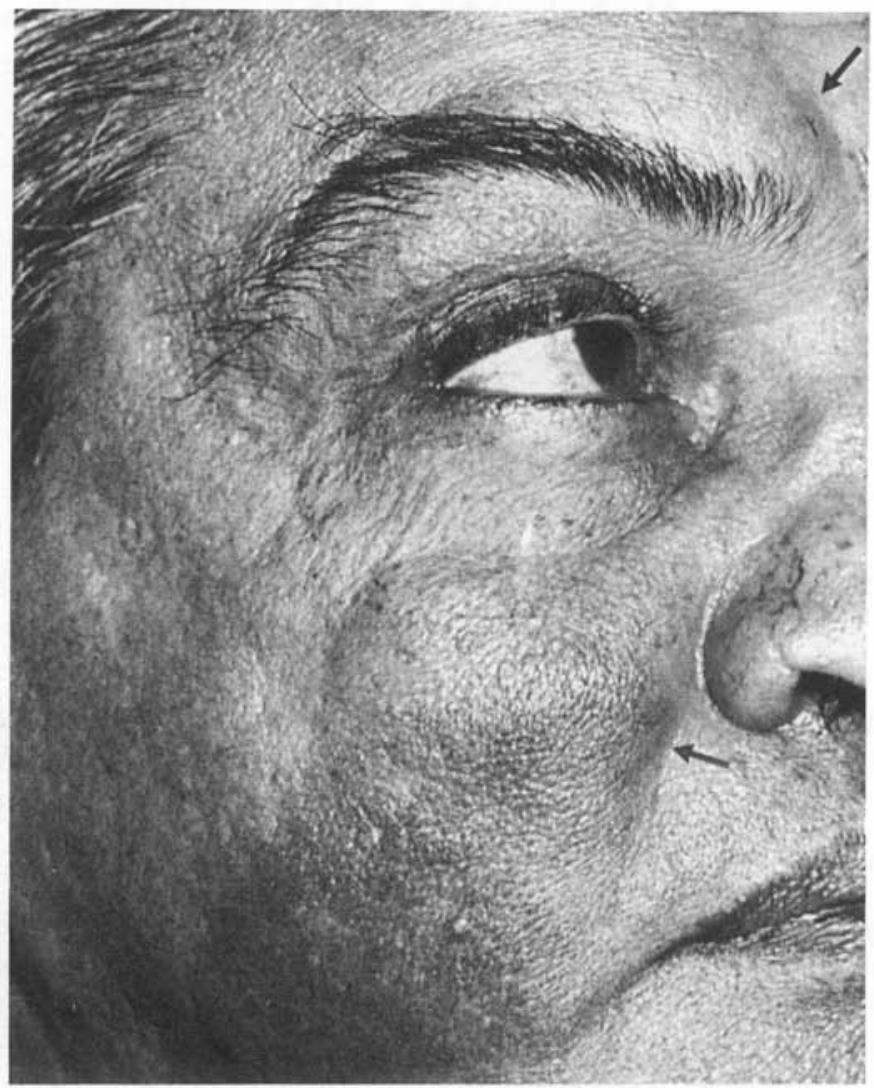

Figure 9. Patient No. 4. Relapse lesions in 1978; raised, sharp-edged plaques (edges arrowed) are present on the face and forehead.

Mitsuda reaction measured $4 \mathrm{~mm}$, but histologically it showed the same tuberculoid but rather non-specific features as the skin lesion. Peripheral blood lymphocytes responded positively to stimulation with $M$. leprae (Dr G A W Rook). An attempt to isolate his strain of $M$. leprae was unsuccessful.

The facial lesions slowly settled with rifampicin and ethionamide, becoming completely inactive after 16 months. In 1988, his lepromin Mitsuda reaction measured $5 \mathrm{~mm}$, a biopsy showing epithelioid cell granulomas (Dr S Lucas). He died of intercurrent disease.

\section{PATIENT SN}

This patient, aged 29, was classified as lepromatous in Cyprus in 1950, his skin smears being strongly positive. He commenced treatment with solapsone sodium and thiacetazone. On referral to the Hospital for Tropical Diseases in 1953, he was noted to have a resolving leonine facies, prominent eye brows and no rash or nodules. Although smears contained only 'scanty bacilli', histology revealed several masses of globi and he was classified by Dr R G Cochrane as resolving leproma. This was confirmed by subsequent 


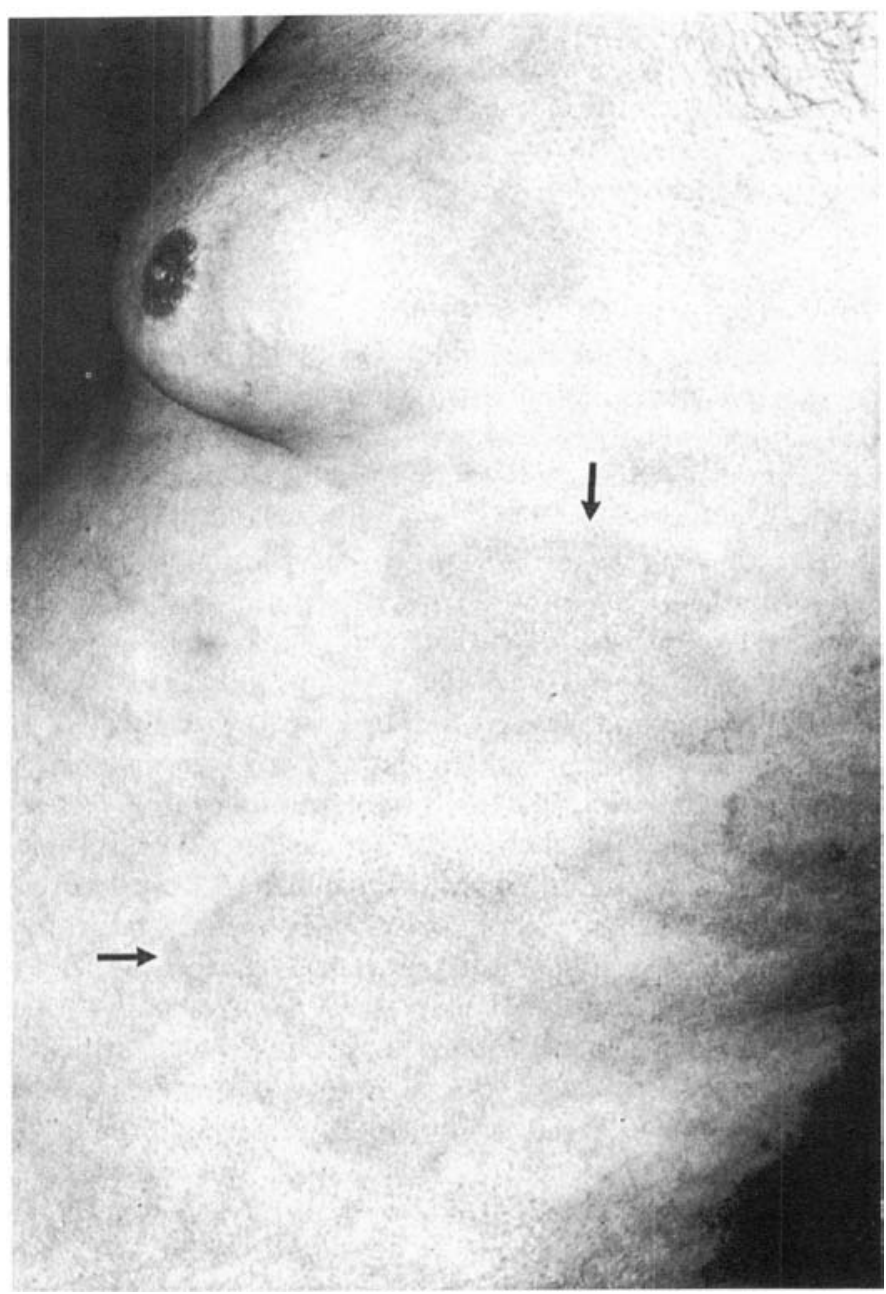

Figure 10. Patient No. 5. Right flank in 1984, showing dry erythematous plaques (edges arrowed) consistent with BT leprosy. Note the gynaecomastia.

re-examination of the section, which showed a resolving lesion with many lymphocytes, being classified LLs, almost BL. Dapsone therapy was continued, but another biopsy in 1956 was more active. He suffered from ENL in 1957 and became smear negative in 1960. His smears became weakly positive in 1975, and his dose of dapsone, previously around $50 \mathrm{mg}$ twice weekly for many years, was raised to $50 \mathrm{mg}$ and then $100 \mathrm{mg}$ daily. Nevertheless, several urine tests were negative for dapsone in 1978-79, although subsequently compliance improved. His smears were negative again in 1983. He remained clinically quiescent until June 1984, when erythematous anaesthetic macules and plaques developed on his back and chest, which over the course of weeks faded and then relapsed (Figure 10). Smears from the new lesions were negative (left elbow site was $1+$ ). Histological examination revealed typical BT leprosy, quiescent or mildly active, with fairly numerous lymphocytes and scanty granular AFB. There was no sign of reaction and 
the lesions appeared to be in a moderately late stage of relapse with upgrading. His Mitsuda reaction was negative on two occasions. His treatment was changed to MDT (rifampicin, ethionamide and dapsone) in September 1984, and the skin lesions flattened and faded over the next four months. The Mitsuda reaction, repeated in 1989, remained negative. Organisms obtained prior to MDT were not infectious to mice.

\section{PATIENT DD}

This Cypriot patient was seen at the Hospital for Tropical Diseases in 1954, aged 18. She gave a history of having been treated with injections for leprosy for eight years. On examination, she had signs of early active lepromatous leprosy, with a few small nodules on her face and erythematous areas on her limbs and buttocks; her ears were not enlarged and the eyebrows were intact. Her BI was $4 \cdot 0$, with solid-staining bacilli present. Skin biopsy revealed active lepromatous (LLs) leprosy. A lepromin test was negative. She was treated with silver sulphone for six months, and then with dapsone by injection. By 1957 she showed no stigmata of leprosy, and had only one small area of anaesthesia on the foot. She continued treatment with oral dapsone $100 \mathrm{mg}$ daily. Her skin smears became negative in 1959 and the dapsone was reduced to $25 \mathrm{mg}$ twice weekly.

In 1979 there was mild clawing of her left toes, with anaesthesia of the left foot and the lateral side of the left leg. Granular AFB $(2+)$ were found in a finger smear. The Mitsuda reaction was negative. In 1982, when clinically her disease was completely inactive, she agreed to take dapsone $50 \mathrm{mg}$ daily, plus rifampicin $600 \mathrm{mg}$ for two consecutive days each month, but refused clofazimine and ethionamide. Seven months later she began to suffer from neuritic pains, and developed a dry, erythematous plaque over her right index and mid-fingers and the associated part of her right palm, a similar plaque over her left lower chest posteriorly, as well as faint erythematous lesions on the left buttock and both calves. The two plaques were anaesthetic. The dorsal branch of the right superficial terminal ulnar nerve was considerably enlarged, and the right superficial radial and ulnar nerves

had increased in size. Skin smears from the new lesions and earlobes were negative for AFB. Biopsy of the left back plaque revealed a small BT lesion with signs of reaction. Scanty $(2+)$ granular AFB were present in a nerve bundle. Her Mitsuda reaction measured $3 \mathrm{~mm}$ at four weeks. It was thought that she had developed a very early subclinical relapse, probably due to dapsone resistance, before commencing rifampicin and standard dapsone therapy, and that a reversal reaction had occurred after seven months. She was treated with prednisolone, chemotherapy was continued, including ethionamide which she now accepted, and the lesions became quiescent after 18 months.

Table 1 summarizes the time scale and salient points of the six patients.

\section{Discussion}

We submit that all six patients were suffering from subpolar lepromatous (LLs) leprosy when first they commenced treatment with effective chemotherapy. Patients 1 and 2 showed the stigmata of old LL leprosy and their classification is confirmed by the time taken to become skin smear negative. The remaining four patients all had their classification confirmed histologically. All became bacteriologically negative and, at the time of their relapses, five of the six had been negative on skin smear for 14 years or more.

It is reasonable to suppose that relapse was accounted for by dapsone resistance in all 
Table 1. Details of the six lepromatous patients, timing, histological classification, lepromin results and dapsone sensitivity at time of relapse

\begin{tabular}{|c|c|c|c|c|c|c|c|c|}
\hline \multirow[b]{2}{*}{$\begin{array}{l}\text { Patient } \\
\text { number }\end{array}$} & \multicolumn{2}{|c|}{ Year } & \multicolumn{2}{|l|}{ Relapse } & \multicolumn{2}{|c|}{ Relapse (years) } & \multirow[b]{2}{*}{$\begin{array}{l}\text { Dapsone } \\
\text { resistance }\end{array}$} & \multirow[b]{2}{*}{$\begin{array}{l}\text { Lepromin (Mitsuda) } \\
\text { reading at relapse }\end{array}$} \\
\hline & $\begin{array}{l}\text { of commencing } \\
\text { sulphone therapy }\end{array}$ & $\begin{array}{c}\text { skin smears } \\
\text { became negative }\end{array}$ & histology & year & $\begin{array}{l}\text { since starting } \\
\text { sulphones }\end{array}$ & $\begin{array}{c}\text { since smears } \\
\text { became negative }\end{array}$ & & \\
\hline $1 \quad(5077)$ & 1948 & 1959 & BT Mild Reaction & 1975 & 27 & 16 & Proven & $6 \mathrm{~mm}$ (ulcerated) \\
\hline $2 \quad(8715)$ & 1950 & 1960 & BT Reaction & 1974 & 24 & 14 & Proven & $5 \mathrm{~mm}$ \\
\hline 3 (RS) & 1966 & 1973 & BT & 1977 & $11 \frac{2}{12}$ & $3 \frac{8}{12}$ & Proven & $3 \mathrm{~mm}$ \\
\hline 4 (TB) & 1950 & 1956 & Ind/Tuberculoid & 1978 & 28 & 22 & Prima facie & $4 \mathrm{~mm}$ (histology BT) \\
\hline $5 \quad(\mathrm{SN})$ & 1950 & 1960 & BT mildly active & 1984 & 34 & 24 & Prime facie & 0 \\
\hline 6 (DD) & 1954 & 1959 & BT Reaction & 1983 & 28 & 23 & Prima facie & $3 \mathrm{~mm}$ \\
\hline
\end{tabular}


cases. In the first three patients, resistance was proved by the mouse footpad technique. It is noteworthy that it took up to four years after the initial relapse to obtain a satisfactory inoculum; and in the two subsequent patients mouse footpad inoculation of their strains of $M$. leprae was unsuccessful, mainly because it was considered unethical indefinitely to delay changing treatment. Patent No. 6 had already commenced treatment with rifampicin before her clinical relapse and isolation of her strain of $M$. leprae was not attempted. Patients Nos 4, 5 and 6 had commenced chemotherapy with early, substituted sulphones, and Meade et al. ${ }^{7,8}$ reported that the risk of relapse from dapsone resistance was three times greater in lepromatous patients commencing treatment with solapsone than with dapsone in full dosage. The incubation period of up to 34 years presents no problem; we have observed patients relapse with proven dapsone resistance as late as 33 and 37 years after commencing sulphone therapy. ${ }^{9}$

In our experience, the majority of lepromatous patients are histologically of the subpolar LLs type that have downgraded from BB or even BT. ${ }^{10}$ Observations in the presulphone era suggest that perhaps $5 \%$ of BT patients downgraded across the spectrum. ${ }^{11}$ Therefore it is surprising that reversion to BT on relapse has not been reported earlier. The account of the development of BT lesions, 12 years af ter commencing sulphones in a 'borderline-lepromatous' patient reported in $1962^{12}$ (Fernandez et al. case no. 2) is suggestive of a late reversal (upgrading, Jopling type 1) reaction in a LLs patient and not necessarily of a bacterial relapse. The case reported by Jonquieres et al. ${ }^{13}$ in 1963, who stopped sulphone therapy after 11 years because of intercurrent disease and who developed a BT relapse two years later, with a $3 \mathrm{~mm}$ Mitsuda reaction, appears very similar to our third patient. But very late relapses with Mitsuda conversion do not appear to have been reported.

The observation that relapse might follow the restarting (case 3) or increasing (case 6) of chemotherapy suggests that chemotherapy may help to uncover, or make clinically apparent, a relapse. Similarly tuberculoid leprosy may follow prophylactic sulphones. ${ }^{14,15}$ In case 3, however, a biopsy had shown the evolution of certain tuberculoid features 21 months before the clinical onset of relapse. This was associated with slight histological reaction and one short solid bacillus. It seems probable that in these very long treated patients, a clinically silent upgrading had taken place that might never have been detected but for the accident of a relapse. But it cannot be excluded that late stage relapse might help to trigger upgrading after the manner of a vaccine.

The role of reactions in the upgrading process may be questioned. There were no overt reactions, although in one case there were neuritic pains. Histological signs of reaction were reported in three cases, but they were mild and more in the nature of incipient reactions which are often clinically silent. There was some enhancement of delayed hypersensitivity, as indicated by the development of Mitsuda reactivity, and the low level of reaction in the lesions is probably explained by the low level of mycobacterial antigen present. It is noteworthy that five of our six patients developed their BT relapses 24 to 34 years after commencing effective chemotherapy, and 14 to 24 years after becoming smear negative. As discussed in our associated paper, ${ }^{5}$ we consider that residual antigen is only slowly removed from the dermis and may persist for many years (perhaps 10 or more) after an LL patient has become negative for AFB by slit-skin smear examination. During this time a patient might be expected to remain lepromin negative. The relapses which we report appeared, both clinically and histologically, to follow a course similar to that of an initial infection prior to the development of delayed hypersensitivity: even the Mitsuda 
reaction in one of the two cases that were biopsied showed a predominantly lymphocytic response. Such relapse, with limited bacterial multiplication in almost resolved lesions, would increase the ratio of dermal to systemic antigen, since any residual antigen from old lesions would, after this duration of time, have mostly drained to the reticulo-endothelial system. It would also increase the ratio of live to dead bacilli in the host, and the ratio of surface and secreted antigen to exposed cytoplasmic antigen. If residual antigen is, as we suggest, quantitatively minute in such long-term treated patients, two of these three factors would also apply to their lepromin tests and could account for the finding of positive (3 $\mathrm{mm}$ or greater) Mitsuda lepromin reactions.

If this explanation is correct, we might expect that many similarly long-term treated LL patients, with no history of relapse, might also with time become lepromin positive, as indeed we have found. ${ }^{5}$ Nevertheless, it is clear from the five of our six relapse patients who became lepromin positive, that the development of a positive Mitsuda test is, of itself, no test of cure in LL leprosy, nor necessarily an indication of adequate protective immunity.

\section{Acknowledgments}

We particularly thank Dr W H Jopling for access to his meticulous clinical notes of patients 3-6, and Dr A B G Laing for supplying follow-up details of patient no. 1. We also thank Drs R J W Rees and M J Colston for attempting to isolate strains of M. leprae from three of the patients. The Leprosy Research Unit, Sungai Buluh, where we studied patients Nos 1 and 2, was (until 1981) jointly sponsored by the Malaysian Ministry of Health and the British Medical Research Council.

\section{References}

1 Pearson JMH, Rees RJW, Waters MFR. Sulphone resistance in leprosy. A review of one hundred proven clinical cases. Lancet, 1975; 2: 69-72.

2 Waters MFR. The diagnosis and management of dapsone-resistant leprosy. Lepr Rev, 1977; 48: 95-105.

3 Waters MFR, Ridley DS. Tuberculoid relapse in lepromatous leprosy. Int J Lepr, 1979; 47: 350.

${ }^{4}$ Ridley DS. Histological classification and the immunological spectrum of leprosy. Bull WHO, 1974; 51: 45165 .

5 Waters MFR, Ridley DS, Lucas SB. Positive Mitsuda lepromin reactions in long-term treated lepromatous leprosy. Lepr Rev, 1990; 61: 347-52.

6 Waters MFR, Rees RJW, Laing ABG, Khoo Kah Fah, Meade TW, Parikshak N, North WRS. The rate of relapse in lepromatous leprosy following completion of twenty years of supervised sulphone therapy. Lepr Rev, 1986; 57: 101-9.

7 Meade TW, Pearson JMH, Rees RJW, North WRS. The epidemiology of sulphone-resistant leprosy. Int $J$ Lepr, 1973; 41: 684 .

${ }^{8}$ Pearson JMH. The problem of dapsone-resistant leprosy. Lepr Rev, 1981; 49: 417-20.

9 Waters MFR. The chemotherapy of leprosy. In: The Biology of the Mycobacteria, volume 3. Ratledge C, Stanford JL, Grange J (eds), London: Academic Press, 1989, Ch 9, 405-74.

10 Ridley DS, Waters MFR. Significance of variations within the lepromatous group. Lepr Rev, 1969; 40: 14352 .

11 Nousitou F. Some aspects of tuberculoid leprosy and chemotherapeutic trials. Acta Leprologia (Genève), 1979; 74: 1-32.

12 Fernandez JMM, Carboni EA, Mercau RA, Sereal A. Transformation of two borderline-lepromatous leprosy cases to tuberculoid with healing. Int J Lepr, 1962; 30: 254-65.

13 Jonquieres EDL, Melamed AJ, Manzi RO. Tuberculoid relapse in a healed lepromatous (L3) case. Int J Lepr, 1963; 31: 1-8.

14 Noordeen SK. Chemoprophylaxis of leprosy. Lepr India, 1969; 61: 247-54.

15 Russell DA, Worth RM, Jano B, Fasal P, Shepard CC. Acedapsone in the prevention of leprosy: field trial in three high prevalence villages in Micronesia. Am J Trop Med Hyg, 1979; 28: 559-63. 